\title{
How critical is frame selection in quantitative coronary angiographic studies?
}

\author{
J. H. C. Reiber, P. van Eldik-Helleman, C. J. Kooijman, J. G. P. Tiussen and \\ P. W. Serruys \\ Thoraxcenter, Erasmus University and University Hospital Dijkzigt, Rotterdam, \\ The Netherlands
}

KEY WORDS: Quantitative coronary arteriography, validation study, frame selection.

To analyse quantitatively a coronary arterial segment from a cineangiogram, an end-diastolic or neighbouring frame is usually selected. However, different cardiologists may select other (although usually neighbouring) frames, even when the same selection criteria are followed. It is also possible that the frames are selected from different cardiac cycles. In this study the effects of such phase shifts on the reproducibility of the quantitative measurements were studied.

In a total of 38 consecutive patient films obtained at a filmspeed of 25 frames $s^{-1}$, the frame $\phi$ demonstrating the severity of a lesion optimally as judged by a senior cardiologist, the three preceding frames, the three following frames and one frame exactly one cycle prior to or following frame $\phi$ were selected; frame $\phi$ was always chosen in the end-diastolic phase of the cardiac cycle. In each film one coronary arterial segment with a focal lesion was analysed quantitatively in these total of 8 frames with the Cardiovascular Angiography Analysis System (CAAS).

No significant differences were found in the mean difference and the standard deviations of the differences (variabilities) in the obstruction diameter, interpolated reference diameter, percent diameter stenosis, extent of the obstruction and area of atherosclerotic plaque obtained in the various frames with respect to frame $\phi$. Therefore, it may be concluded that the selection of a cineframe for quantitative analysis in the end-diastolic phase of the cardiac cycle is not very critical. It is argued that the quality of mixing of the contrast agent in the arterial segment is a major source of the observed variations; filling artefacts are potentially present in each of the selected frames. For practical purposes, minimal threshold values for the changes in the obstruction parameters based on $95 \%$ confidence levels can be calculated at the individual patient level and at the group level for the exclusion or inclusion of possibly occurring changes in coronary vasomotor tone.

\section{Introduction}

Quantitative coronary cineangiography is an excellent tool for the assessment of the effects of vasoactive drugs ${ }^{[1-3]}$. Usually, an end-diastolic cineframe is selected for the quantitative analysis of a coronary obstruction. However, if the obstruction is not optimally visible in this particular frame, for example due to overlap with another vessel, a neighbouring frame in the sequence may be selected. Also, an aortic pressure signal or ECG-marker on the cinefilm

Address for correspondence: Johan H. C. Reiber, Erasmus University, Laboratory for Clinical and Experimental Image Processing, C.V.R., EE 2328, P.O. Box 1738, 3000 DR Rotterdam, The Netherlands. may not be available for the optimal selection of the end-diastolic frame; as a result, the visually selected cineframe may not be the true enddiastolic frame.

In addition, different cardiologists may select different (although usually neighbouring) frames, even when the same selection criteria are followed. It is also possible that the frames would be selected from different cardiac cycles relative to the injection time. This uncertainty in the frame selection process raises the question what the effect of the selection of a cineframe at another point in the cardiac cycle may be on the quantitative results. The uncertainty in the frame selection is also present when corresponding frames in pre- and post-intervention angiographic 
studies, such as pre- and post-administration of a vasoactive drug, must be selected.

Theoretically, there are many variables that may influence the measurement of the arterial dimensions from cinefilm ${ }^{[4,5]}$. Spears et al. have found that particularly one variable of practical importance, the contrast medium concentration, has a statistically significant effect on these measurements ${ }^{[6]}$.

In this particular study we were interested in determining the measurement variability in the obstructive arterial dimensions if different frames were proposed for the quantitative analysis in the end-diastolic phase of the cardiac cycle. We assume that the two images to be compared do not differ by more than three cineframes. In addition, we studied the variability in the measurements if the two frames were selected in two subsequent cardiac cycles at exactly the same phase in the cycle. Multi-frame averaging was not applied in our study.

\section{Methods}

QUANTITATIVE CORONARY ARTERIOGRAPHY

The cinefilms were analysed with the Cardiovascular Angiography Analysis System (CAAS), which has been described in detail elsewhere ${ }^{[4,7,8]}$. Eleven of the total of 38 cinefilms were analysed with the Research CAAS-system, while the other 27 cinefilms were analysed with the commercial version of the CAAS, the Pie Data* CAAS. The hardware of the two systems is different, but the application software is identical.

To analyse the dimensions of a coronary arterial segment quantitatively, the following steps need to be performed by the application software packages: (1) computation of the calibration factor on the basis of the contrast catheter; (2) automated boundary detection of the arterial segment; (3) computation of the diameter function from the detected and pincushion-corrected contour positions; and (4) determination of the severity of a coronary obstruction in terms of absolute and relative parameters. A typical example of the results of the quantitative analysis of a coronary obstruction is presented in Fig. 1.

\section{PATIENT MATERIAL}

To study the possible dependency of the obstruction measurements on the selected

* Pie Medical B.V., Maastricht, the Netherlands. image, 38 consecutive patient films were analysed with the CAAS systems. In all cases the filmspeed was 25 frames s$^{-1}$ and the images were obtained with $7^{\prime \prime}$ image intensifiers. A modern, nonionic contrast agent (Iopamidol ${ }^{\circledR}$ ) with low osmolality was used.

The selection of the frame judged optimal for quantitative analysis was performed by one senior cardiologist according to the following criteria: Choose from the end-diastolic frame or a neighbouring frame, the optimal frame that satisfies the following criteria: (1) the obstruction if free of overlap of sidebranches or other structures; (2) maximal sharpness of the vessel boundaries is observed; (3) the obstruction is observed in its most severe appearance.

This selected frame was defined as frame $\phi$. For the purposes of this study, we selected in addition three frames preceding frame $\phi$, denoted frames $-1,-2$ and -3 , and three frames immediately following frame $\phi$, denoted frames $+1,+2,+3$.

To be able to judge the variabilities that will be observed between these frames against the reproducibility of assessing a stenosis in exactly the same phase of the cardiac cycle, an eighth frame was selected exactly one cardiac cycle earlier or later than the optimally selected frame. Due to the differences in contrast filling of the coronary segment, in seven cases the frame was selected exactly one cycle prior to the selected frame (defined as $-1 \mathrm{c}$ ), in 25 cases exactly one cycle following frame $\phi$ (defined as $+1 c)$, while in the remaining six cases an appropriate frame exactly one cycle before or after the selected images could not be found.

The calibration for this series of eight measurements per patient film was performed only once, since the geometry of the X-ray system with respect to the patient remained unchanged.

The 38 coronary segments analysed were RCA-prox (4), RCA-mid (13), RCA-dist (2), LAD-prox (2), LAD-mid (10), Cx-prox (4) and Cx-dist (3).

\section{STATISTICAL ANALYSIS}

The variabilities in the measurements assessed from the frames other than frame $\phi$ were defined by the standard deviations (s.d.) of the differences between these measurements and those from frame $\phi$. Student's $t$-test for paired values was applied to determine the statistical 


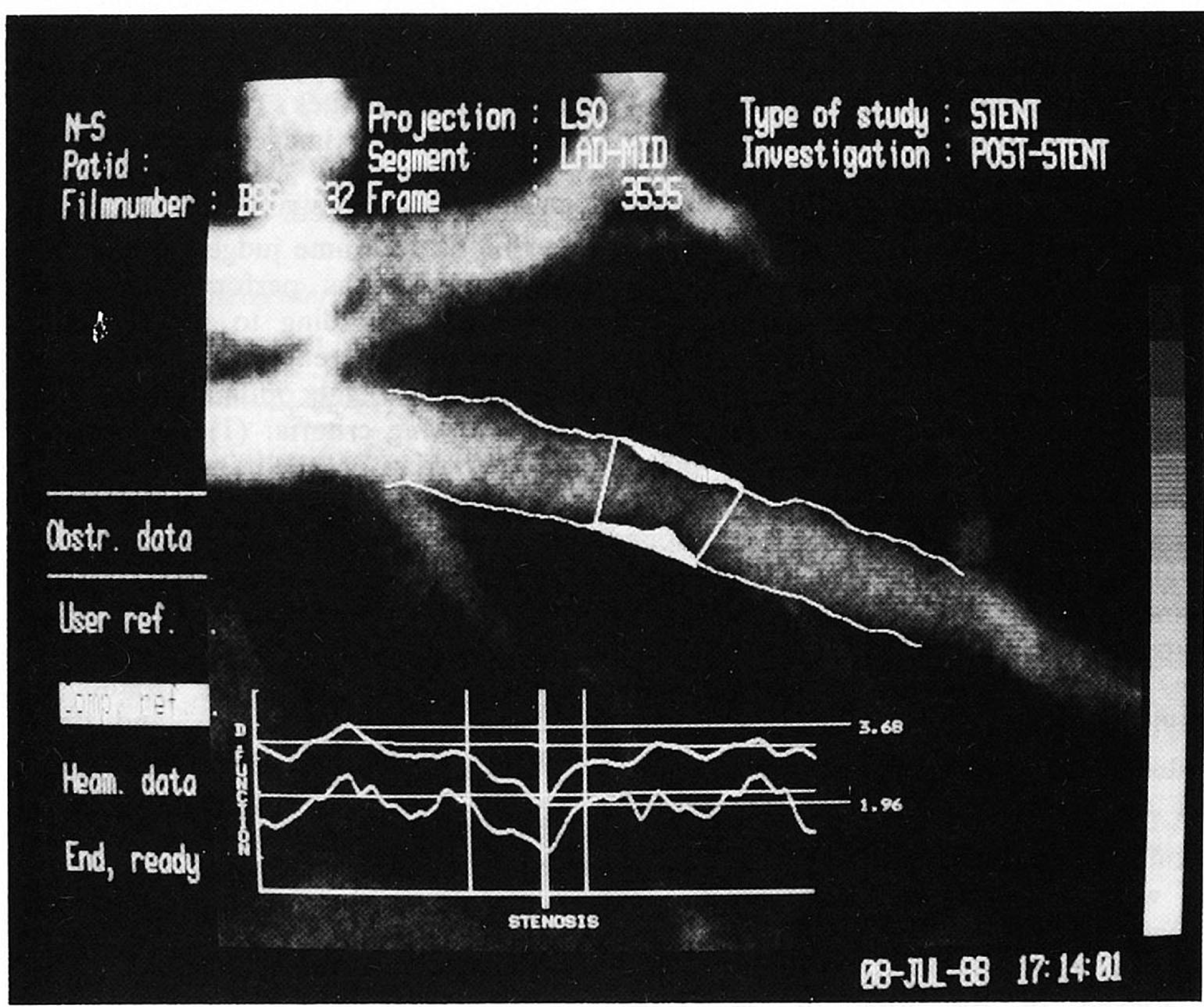

Figure 1 Example of the quantitative analysis of a mid LAD-segment with the CAAS. The contours were detected automatically; absolute and relative parameters can be derived from the diameter function (upper function) and from the densitometric area function (lower function). The parameters investigated in this study were all derived from the diameter function.

significance between the measurements (border of significance, $P=0 \cdot 01)$.

\section{Results}

The parameters compared were the obstruction diameter $(\mathrm{mm})$, interpolated reference diameter $(\mathrm{mm})$, interpolated percent diameter stenosis (\%), extent of the obstruction (mm) and area of atherosclerotic plaque $\left(\mathrm{mm}^{2}\right)$ in a particular view. The results are presented in Table 1. The ranges of the mean differences and standard deviations of the differences of the measurements in the various frames with respect to those in frame $\phi$ have been summarized in Table 2 .

Table 1 Mean differences and standard deviations (mean $\pm s . d$.) of the measurements in the frames preceding the optimal frame by 3, 2 or 1 frame(s) (denoted -3, -2, -1, respectively), in the frames following the optimal frame by 1 , 2 , or 3 frame (s) (denoted $+1,+2,+3$, respectively) or in the frame preceding or following frame $\phi$ by precisely one cardiac cycle $( \pm 1 \mathrm{c})$ with the measurements obtained in frame $\phi$. None of the differences reached statistical significance at the $0 \cdot 01$ level

\section{$-3$}

$-2$

$0 \cdot 02 \pm 0 \cdot 20$

Obstr. diam. (mm) $\quad-0 \cdot 02 \pm 0 \cdot 24$

Ref. diam. (mm)

$\%$-D stenosis (\%)

Extent (mm)

$0 \cdot 03 \pm 0 \cdot 13$

$1 \cdot 07 \pm 7 \cdot 27$

$-0 \cdot 12 \pm 0 \cdot 58$

Area plaque $\left(\mathrm{mm}^{2}\right)$

$0 \cdot 21 \pm 2 \cdot 17$
$-0.71 \pm 5.98-0$.

$0 \cdot 06 \pm 0.54$

$0 \cdot 09 \pm 1.76$
$0 \cdot 02 \pm 0 \cdot 09$

$0 \cdot 02 \pm 0 \cdot 12$

$0.20 \pm 6.46$

$-0.07 \pm 6.45-1$

$0 \cdot 32 \pm 1 \cdot 77$
$0 \cdot 13 \pm 0.47$

$0 \cdot 34 \pm 1 \cdot 94$
$+2$

$+3$

$\pm 1 \mathrm{c}$ 
Table 2 Ranges in the mean differences and standard deviation of the measurements for the 7 frames $(-3,-2$, $-1,+1,+2,+3$ and $\pm 1 c)$ with respect to frame $\phi$, as summarized from Table 1

\begin{tabular}{lcc}
\hline & \multicolumn{2}{c}{ Ranges } \\
\cline { 2 - 3 } & Mean difference & s.d. difference \\
\hline Obstruction diam. (mm) & $0 \cdot 006-0 \cdot 05$ & $0 \cdot 19-0 \cdot 24$ \\
Reference diam. (mm) & $0 \cdot 003-0 \cdot 08$ & $0 \cdot 09-0 \cdot 18$ \\
$\%$-D stenosis $(\%)$ & $0 \cdot 07-1 \cdot 14$ & $5 \cdot 98-7 \cdot 27$ \\
Extent (mm) & $0 \cdot 05-0 \cdot 20$ & $0 \cdot 40-0 \cdot 58$ \\
Area plaque $\left(\mathrm{mm}^{2}\right)$ & $0 \cdot 09-0 \cdot 70$ & $1 \cdot 53-2 \cdot 30$ \\
\hline
\end{tabular}

For all cases the results of the different measurements were not found to be significantly different from the values at frame $\phi$. For the obstruction diameter the mean difference varied from $0.006 \mathrm{~mm}(=6 \mu \mathrm{m})$ to $0.05 \mathrm{~mm}(=50 \mu \mathrm{m})$ with the standard deviation varying from $0 \cdot 19$ to $0.24 \mathrm{~mm}$. For the reference diameter the mean difference values varied from 3 to $80 \mu \mathrm{m}$ with a standard deviation varying from 0.09 to $0.18 \mathrm{~mm}$. Apparently, the reference diameter is more reproducible than the obstruction diameter, which is not surprising if the incomplete mixing of contrast agent is one of the major causes. For the mean differences in the percent diameter stenosis values a lowest value of $0.07 \%$ was found and a highest value of $1 \cdot 14 \%$, while the standard deviations ranged from $5.98 \%$ to $7 \cdot 27 \%$, a very narrow range.

The mean differences in the extent $(\mathrm{mm})$ of the obstruction varied from 50 to $200 \mu \mathrm{m}$ with the standard deviation between 0.40 and $0.58 \mathrm{~mm}$. Finally, for the area of the atherosclerotic plaque the range for the mean differences was found to be 0.09 to $0.70 \mathrm{~mm}^{2}$ with the standard deviations between 1.53 and $2 \cdot 30 \mathrm{~mm}^{2}$.

From all these data no consistent pattern could be found for a particular frame, being characterized by producing smaller values in terms of mean difference or standard deviation than any other frame. Also, the reproducibility of analysing a coronary obstruction one complete cardiac cycle earlier or later than the originally selected cineframe seems to be just as good as taking a neighbouring frame of frame $\phi$.

We propose to define for each quantitative parameter a variability measure due to the frame selection process, which is equal to the maximal value of the seven standard deviation values from Table 1 (Table 3).
Table 3 Variability measures in the assessment of coronary obstruction parameters due to the frame selection process; the value presented for a particular parameter equals the maximal value of the seven standard deviations for that parameter from Table 1

\begin{tabular}{ll}
\hline Obstruction diameter $(\mathrm{mm})$ & $0 \cdot 24$ \\
Reference diameter $(\mathrm{mm})$ & $0 \cdot 18$ \\
$\%$-D stenosis $(\%)$ & $7 \cdot 27$ \\
Extent obstruction $(\mathrm{mm})$ & $0 \cdot 58$ \\
Plaque area $\left(\mathrm{mm}^{2}\right)$ & $2 \cdot 30$ \\
\hline
\end{tabular}

\section{Discussion}

In this study the variability in the assessment of coronary obstruction parameters due to the frame selection process has been assessed in a group of 38 patient studies by analysing three frames before and three frames following a frame $\phi$ demonstrating the severity of a lesion optimally as judged by a senior cardiologist, and one frame exactly one cycle prior to or following frame $\phi$.

The question now becomes Which factors are responsible for the measured variations in this study? First, all the possible variations in the data procedure itself, as discussed in detail in Refs 4 and 5 are present. These variations include: (1) quantum noise in the images; (2) noise contributions in the video or CCD camera; (3) quantitation errors in the analog-to-digital conversion; (4) the effects of resampling the data along scanlines through the square grid of the digital data; (5) observer variations in the definition of centre positions within the selected arterial segment; (6) possible manual corrections to the detected contours; and (7) selection of beginning and end points of the obstructive lesion. However, these factors have been shown to be responsible for variations in the obstruction diameter, reference diameter and \%-D stenosis of only $0.10 \mathrm{~mm}, 0.10 \mathrm{~mm}$ and $3.94 \%$, respectively ${ }^{[4,5]}$. Since the variabilities in this study in general are larger than these values, additional causes in the acquisition procedure must be present.

A factor that certainly plays an important role in the angiographic image acquisition procedure, is the variation in the quality of mixing of the contrast agent with the blood. Other factors in the angiographic procedure mentioned in Refs 4 and 5 , such as differences in the angles and height of the X-ray gantry with respect to the 
patient at the time of repeated angiography, differences in the vasomotor tone of the coronary arteries and deviations in the size of the catheter as listed by the manufacturer from the true size do not apply in this particular comparative study, since for each patient study different frames from the same angiographic investigation were análysed. However, one additional factor must be taken into account, representing the variability in the size and shape of the coronary arterial segment from frame-toframe; size changes may be caused by foreshortening of the vessel segment. In general, the variation due to this last factor will be small, since the images were selected in the enddiastolic phase, where there is little motion from frame-to-frame.

Finally, it is known from the literature that the dimensions of a normal coronary arterial segment may vary by as much as $6.0 \pm 2.0 \%$ over a cardiac cycle due to the pulsatile effect of the aortic pressure ${ }^{[9]}$. These variations were measured between end-diastole and end-systole. Therefore, the variations over several frames in the end-diastolic phase may be neglected as compared to other existing variations.

If we compare the error sources present in the repeated analysis variability study and those in the present study, then we must conclude that the increase in the variabilities must be due to the following factors: quality of mixing of the contrast agent, the changes in size and shape of the arterial segment and the pulsatile effect. It has been argued above that the last two factors are most probably very small; thus we must conclude that the quality of the mixing of the contrast agent with the blood plays a major role as a source of variation.

Now that these variabilities in the obstruction parameters due to the frame selection procedure have been assessed, one may also wonder whether it is possible to define minimal threshold values for each of these obstruction parameters such that changes in coronary vasomotor tone can be excluded when the changes observed in these obstruction parameters are smaller than these threshold levels. When trying to answer such questions, one should always distinguish between the consequences of the changes measured at the patient level and those at the group level. For example, if one observes in an individual patient a change in obstruction diameter of $0.20 \mathrm{~mm}$ over the
Table 4 Minimal threshold values for the occurrence of vasomotor changes at the individual patient level $(95 \%$ confidence level)

\begin{tabular}{lr}
\hline Obstruction diameter $(\mathrm{mm})$ & $0 \cdot 47$ \\
Reference diameter $(\mathrm{mm})$ & $0 \cdot 35$ \\
$\%$-D stenosis $(\%)$ & $14 \cdot 25$ \\
Extent obstruction $(\mathrm{mm})$ & $1 \cdot 14$ \\
Plaque area $\left(\mathrm{mm}^{2}\right)$ & $4 \cdot 51$ \\
\hline
\end{tabular}

various frames as defined in this study, one is well within the range of possible measurement error $(0.24 \mathrm{~mm})$. As a consequence, one cannot tell whether the measurement in this individual patient represents a true change in coronary vasomotor tone, or just measurement error. This can also be described more quantitatively with the theory of the confidence limits. For the example of an individual change of $0.20 \mathrm{~mm}$, all values of the true changes in obstruction diameter due to variations in coronary vasomotor tone within the interval of $0.20 \pm(1.96 \times$ 0.24 ) being $-0.27,0.67$ are compatible with this observation*. As a consequence, the value 0 (no vasomotion), but also 0.20 is acceptable, but the observation virtually excludes 1.00 as a possibility. In other words, one can say that changes in coronary vasomotor tone are truly present with $95 \%$ confidence, if the individual changes in obstruction diameter exceed $1.96 \times 0.24=$ $0.47 \mathrm{~mm}$. On the other hand, a value below $0.47 \mathrm{~mm}$ does not exclude changes in vasomotor tone; it only indicates that changes in vasomotor tone, if present, cannot be measured with sufficient precision. Similarly, minimal threshold values for changes in the other obstruction parameters at the individual patient level can be calculated (Table 4).

However, if an average change (before and after the intervention) of $0.20 \mathrm{~mm}$ in the obstruction is measured in a series of say, 100 patients, one can be certain that this change reflects more than measurement error alone. Again, with the theory of confidence limits, the $95 \%$ confidence interval for a mean change of $0.20 \mathrm{~mm}$ is $0.20 \pm(1.96 \times 0.24 / \sqrt{100})$ being $[0 \cdot 15,0.25]$, which excludes the value of 0 (no vasomotion).

Thus, using the theory of confidence limits minimal threshold values for the inclusion or exclusion of changes in coronary vasomotor tone

* 1.96 is the 2.5 percentile of the normal distribution. 
can be defined both at the patient and at the group level; at the group level such threshold values will be dependent on the measurement error and on the group size.

In summarizing the results presented in this paper, one may conclude that the selection of a cineframe for quantitative analysis in the end-diastolic phase of the cardiac cycle is not very critical. The variabilities in the measurements are not influenced statistically significant if the selected frame in the same cineangiographic film sequence are out of phase by a maximum of three frames at a film speed of 25 frames $s^{-1}$, or if a frame is selected exactly one cardiac cycle earlier or later. Most probably, the variations in the filling of the arterial segment with the contrast agent are a major source of the variations, being potentially present in each of the selected frames. Other possible error sources in the data acquisition procedure, such as changes in size and shape of the arterial segments, and the pulsatile effect, most probably play a minor role.

Therefore, the results from this study must be very reassuring to those involved in quantitative coronary angiographic studies. They show that reliable quantative data about coronary morphology can be obtained from cinefilm if certain, not extremely demanding, precautions are followed. Furthermore, minimal threshold values for the changes in the obstruction parameters based on $95 \%$ confidence levels can be calculated both at the individual patient level and at the group level for the exclusion or inclusion of possibly occurring changes in coronary vasomotor tone.

The authors wish to thank Mrs B. Smit-van der Deure for her secretarial contributions in the preparations of this manuscript.

\section{References}

[1] Serruys PW, Hooghoudt TEH, Reiber JHC, Slager C, Brower RW, Hugenholtz PG. Influence of intracoronary nifedipine on left ventricular function, coronary vasomotility, and myocardial oxygen consumption. Br Heart J 1983; 49: 427-41.

[2] Serruys PW, Lablanche JM, Reiber JHC, Bertrand ME, Hugenholtz PG. Contribution of dynamic vascular wall thickening to luminal narrowing during coronary arterial vasomotion. Z Kardiol 1983; 72: 116-23.

[3] Deckers JW, Reiber JHC, Serruys PW. Long-lasting vasodilatory effect of intracoronary administration of molsidomine metabolite SIN-1. In: Bing RJ, Stauch $M$, eds. Ischemic heart disease and heart failure. Advances in treatment with molsidomine. Munich/Vienna/Baltimore, Urban and Schwarzenberg, 1986: 80-5.

[4] Reiber JHC, Serruys PW, Kooijman CJ et al. Assessment of short-, medium-, and long-term variations in arterial dimensions from computerassisted quantitation of coronary cineangiograms. Circulation 1985; 71: 280-88.

[5] Reiber JHC, Serruys PW, Kooijman CJ, Slager CJ, Schuurbiers JCH, Boer A den. Approaches towards standardization in acquisition and quantitation of arterial dimensions from cineangiograms. In: Reiber JHC, Serruys PW, eds. State of the art in quantitative coronary arteriography. Dordrecht, Martinus Nijhoff Publishers, 1986: 145-72.

[6] Spears JR, Sandor T. Quantitation of coronary artery stenosis severity: limitations of angiography and computerized information extraction. In: Reiber JHC, Serruys PW, eds. State of the art in quantitative coronary arteriography. Dordrecht, Martinus Nijhoff Publishers, 1986: 103-24.

[7] Reiber JHC, Serruys PW, Slager CJ. Quantitative coronary and left ventricular cineangiography; methodology and clinical applications. Dordrecht, Martinus Nijhoff Publishers, 1986.

[8] Reiber JHC, Kooijman CJ, Slager CJ et al. Taking a quantitative approach to cine angiogram analysis. Diagnostic Imaging 1985; 7: 87-9.

[9] Hori M, Inoue $M$, Shimazu $T$ et al. Clinical assessment of coronary arterial elastic properties by the image processing of coronary arteriograms. Comp Cardiol 1983; 393-95. 